\title{
Determinants of elevated chemerin as a novel biomarker of immunometabolism: data from a large population-based cohort
}

\author{
Liselot Koelman ${ }^{1,2}$, Robin Reichmann ${ }^{2,3}$, Claudia Börnhorst' ${ }^{4}$, Matthias B Schulze ${ }^{1,2,5}$, Cornelia Weikert ${ }^{6,7}$, \\ Ronald Biemann ${ }^{8}$, Berend Isermann ${ }^{8}$, Andreas Fritsche ${ }^{9}$ and Krasimira Aleksandrova ${ }^{3,10}$ \\ ${ }^{1}$ Department of Molecular Epidemiology, German Institute of Human Nutrition Potsdam-Rehbruecke (DIfE), Nuthetal, Germany \\ ${ }^{2}$ Institute of Nutritional Science, University of Potsdam, Potsdam, Germany \\ ${ }^{3}$ Department of Epidemiological Methods and Etiological Research, Leibniz Institute for Prevention Research and Epidemiology - BIPS, Bremen, Germany \\ ${ }^{4}$ Department of Biometry and Data Management, Leibniz Institute for Prevention Research and Epidemiology - BIPS, Bremen, Germany \\ ${ }^{5}$ German Center for Diabetes Research (DZD), Neuherberg, Germany \\ ${ }^{6}$ Department of Food Safety, German Federal Institute for Risk Assessment, Berlin, Germany \\ ${ }^{7}$ Institute for Social Medicine, Epidemiology and Health Economics, Charité University Medical Center, Berlin, Germany \\ ${ }^{8}$ Institute of Laboratory Medicine, Clinical Chemistry and Molecular Diagnostics, University of Leipzig, Leipzig, Germany \\ ${ }^{9}$ Division of Endocrinology, Diabetology, Nephrology, Vascular Disease and Clinical Chemistry, Department of Internal Medicine, University of Tübingen, \\ Tübingen, Germany \\ ${ }^{10}$ Faculty of Human and Health Sciences, University of Bremen, Bremen, Germany
}

Correspondence should be addressed to K Aleksandrova: aleksandrova@leibniz-bips.de

\begin{abstract}
Objective: Chemerin is a novel inflammatory biomarker suggested to play a role in the development of metabolic disorders, providing new avenues for treatment and prevention. Little is known about the factors that predispose elevated chemerin concentrations. We therefore aimed to explore a range of lifestyle-associated, dietary, and metabolic factors as potential determinants of elevated chemerin concentrations in asymptomatic adults. Design: We used cross-sectional data from a random subsample of 2433 participants (1494 women and 939 men) aged 42-58 years of the European Prospective Investigation into Cancer and Nutrition-Potsdam cohort.

Methods: Random forest regression (RFR) was applied to explore the relative importance of 32 variables as statistical predictors of elevated chemerin concentrations overall and by sex. Multivariable-adjusted linear regression was applied to evaluate associations between selected predictors and chemerin concentrations.

Results: Results from RFR suggested BMI, waist circumference, C-reactive protein, fatty liver index, and estimated glomerular filtration rate as the strongest predictors of chemerin concentrations. Additional predictors included sleeping duration, alcohol, red and processed meat, fruits, sugar-sweetened beverages (SSB), vegetables, dairy, and refined grains. Collectively, these factors explained $32.9 \%$ variation of circulating chemerin. Multivariable-adjusted analyses revealed linear associations of elevated chemerin with metabolic parameters, obesity, longer sleep, higher intakes of red meat and SSB, and lower intakes of dairy.

Conclusions: These findings come in support of the role of chemerin as a biomarker characterizing inflammatory and metabolic phenotypes in asymptomatic adults. Modifiable dietary and lifestyle-associated determinants of elevated chemerin concentrations require further evaluation in a prospective study setting.
\end{abstract}

\author{
Key Words \\ - chemerin \\ - inflammation \\ - biomarkers \\ - lifestyle determinants
}

Endocrine Connections (2021) 10, 1200-1211

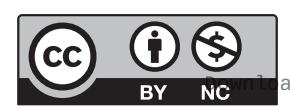

This work is licensed under a Creative Commons Attribution-NonCommercial 4.0 International License. ded from Bioscientifica.com at 04/26/2023 09:43:50AM 


\section{Introduction}

Over the past decade, chemerin has been increasingly implicated as a biomarker of immunometabolism, linking low-grade inflammation and metabolic disorders (1). Chemerin was first discovered as a chemokine with strong chemoattractant activity on various immune cells, including macrophages, dendritic cells, and natural killer cells with key roles in both innate and adaptive immunity (2). It was further rediscovered as an adipokine that is expressed in the adipocytes and was shown to be associated with obesity, insulin resistance, inflammation, and fatty liver disease (FLI) $(3,4)$. Experimentaland clinical data suggested thatcirculating chemerin levels are associated with chronic diseases, such as cardiovascular disease (5) including atherosclerosis (6) and chronic heart failure (7). In addition, chemerin was associated with risk of colorectal cancer (8) and all-cause mortality (9). Thus, chemerin could serve either as an early marker or as an independent predictor of chronic subclinical inflammation. To guide strategies for precision prevention, better understanding of the factors that predispose elevated chemerin concentrations is highly warranted.

The evidence, however, on the potential influence of dietary and lifestyle factors on circulating chemerin has been scant. Several small trials investigated specific interventions for weight loss (hypocaloric diet and increased physical activity) in reducing chemerin levels $(10,11,12)$. In addition, a few observational studies explored associations between chemerin and dietary patterns (13), physical activity (14), and selected health behaviors, that is, smoking and alcohol consumption (15). So far, large observational studies that simultaneously evaluate multiple lifestyle factors in relation to chemerin concentrations are lacking.

We therefore aimed to identify main statistical predictors as potentially important determinants of elevated chemerin concentrations among a wide range of lifestyleassociated, dietary, and metabolic factors and to characterize the associations between selected determinants and elevated chemerin concentrations using data from a large population sample within the European Prospective Investigation into Cancer and Nutrition (EPIC)-Potsdam cohort.

\section{Material and methods}

\section{Study population}

EPIC-Potsdam is a prospective cohort study intended to investigate the role of diet in the development of chronic diseases (16). From 1994 to 1998, 27,548 participants from Potsdam, Germany, and the surrounding geographical area were recruited. For the current analysis, 2500 participants were randomly selected. After exclusions due to unavailable blood samples, implausible dietary intakes (women: $<600$ kcal or $>3500 \mathrm{kcal}$; men: $<800 \mathrm{kcal}$ or $>4200 \mathrm{kcal})$, or outliers, 2433 participants (1494 women and 939 men) remained eligible for this cross-sectional analysis (see Fig. 1 for flow chart). Detailed information about recruitment procedures has been reported elsewhere (17). The study protocol was approved by the Medical Society of the State of Brandenburg, Germany, and all participants provided written-informed consent prior to enrollment (16).

\section{Data collection}

Anthropometric (BMI, waist circumference (WC)) and blood pressure measurements were conducted according to standardized protocols as previously reported (16). Information on socio-demographic characteristics and lifestyle factors, that is, smoking, physical activity, sleeping habits, alcohol consumption, medication, self-reported health satisfaction, and prevalent diseases, were collected using computer-assisted personal interviews. Participants were considered hypertensive at study baseline if they had a systolic blood pressure $\geq 140 \mathrm{mmHg}$, diastolic blood pressure $\geq 90 \mathrm{mmHg}$, reported prior diagnosis of hypertension, or current antihypertensive medication use. Habitual dietary intakes of 12 months prior to recruitment were assessed through validated 148-item semi-quantitative food frequency questionnaires (18). The analyses focused on major food groups shown to be associated with metabolic phenotypes and chronic diseases (19), that is, red and processed meat, fish, dairy products, eggs, vegetables, fruits, nuts, legumes, whole grains, refined grains, and sugarsweetened beverages (SSB). Intake values of food groups and alcohol are presented in grams per day (g/day).

\section{Biomarker measurements}

Participants provided $30 \mathrm{~mL}$ peripheral venous blood at the examination center during daytime hours, which was processed and subsequently stored in tanks of liquid nitrogen at $-196^{\circ} \mathrm{C}$ or in deep freezers at $-80^{\circ} \mathrm{C}$ until time of analysis. Chemerin was measured in citrate-treated plasma with a commercially available sandwich ELISA (BioVendor, Brno, Czech Republic) at the Institute for Clinical Chemistry and Pathobiochemistry, Otto-vonGuericke University Magdeburg (Magdeburg, Germany). Coefficients of variation reported by the manufacturer 




\section{Figure 1}

Flow chart of the study population selection including exclusion and removal of outliers. were 5.1 and $7.0 \%$ within assays and 6.9 and $8.3 \%$ between assays. Chemerin measurements showed good reproducibility over 4 months (intraclass correlation coefficient assessing intra-individual variation: 0.72 (95\% CI: 0.65-0.78)), indicating that one-time chemerin measurements are reasonably representative of the average individual concentration over time (20). Plasma concentrations of cholesterol, triglycerides, HDL-C, high-sensitivity C-reactive protein (hs-CRP), creatinine, $\gamma$-glutamyl transferase (GGT), alanine transaminase (ALT), and uric acid were measured at the Department of Internal Medicine, University of Tübingen (Tübingen, Germany) with an automatic ADVIA 1650 analyzer (Siemens Medical Solutions, Erlangen, Germany) in 2007 (21). All biomarker measurements conducted in plasma were corrected for the dilution introduced by citrate volume to improve comparability with concentrations measured in EDTA-plasma reported in the literature (22). LDL-C was calculated using Friedewald's formula (23). Glomerular filtration rate (eGFR), an indicator of kidney function, was estimated using measured creatinine concentrations based on the proposed formula by the Chronic Kidney Disease Epidemiology Collaboration (24). The fatty liver index (FLI) was used as a proxy of liver fat accumulation (25). Blood draw, sample handling, and laboratory measurements were conducted by experienced technical personnel and followed manufacturer's instructions. Missing biomarker entries $(n=444)$ were imputed for $n=223$ participants (Supplementary Table 1, see section on supplementary materials given at the end of this article) using a random forest procedure within the R package 'missForest' (26). Sensitivity analysis without imputation of biomarkers revealed no differences in effect estimates of main analyses (data provided in Supplementary Table 2).

\section{Statistical analysis}

In descriptive analysis, the distribution of lifestyle-associated, dietary, and metabolic parameters as medians (interquartile

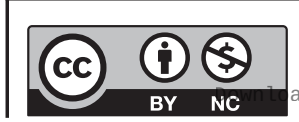


ranges (IQR)) or numbers (percentages) was characterized according to quartiles of chemerin concentrations. Dietary and metabolic variables were modeled as continuous variables along with BMI, WC, recreational sports (hours per week), and sleeping duration (hours of sleep per 24 hours). To reduce the potential effect of multicollinearity between BMI and WC, WC was regressed on BMI to obtain residual WC, which was used in the adjustment models. Alcohol consumption in grams per day was divided by 5 to represent intake per 5 grams. Smoking was modeled as a dichotomized variable (ever smoker vs never smoker). Health satisfaction was modeled as a four-level categorical variable (very dissatisfied to very satisfied). For descriptive analyses, alcohol consumption and sleeping duration were further categorized using predefined cut points $(27,28)$, and physical activity was dichotomized into inactive vs active.

To determine the best set of determinants (statistical predictors) of elevated chemerin concentrations, random forest regression (RFR) was applied as a machine learning technique suited for evaluation of multiple interrelated predictors (29). An important advantage of RFR is the ability to account for potential non-linear association among predictor and response variables. In this analysis, 1000 regression tree models were generated and combined with up to five unique datapoints in each terminal node. The main predictors among the various predictor variables were explored separately (according to exposure profiles - lifestyleassociated factors, dietary factors and metabolic factors) and conjointly and plotted according to relative statistical importance using the R package 'randomForestSRC' (30). In addition, the explained variance of each individual predictor, a set of predictors selected by RFR, as well as all predictors conjointly were estimated using linear regression analyses.

Since RFR only provides information on the relative statistical importance of predictor variables out of a set of variables, but do not show effect size and direction of associations, multivariable-adjusted linear regression was further applied to allow detailed assessment of the associations. In these analyses, food groups and metabolic (clinical/biochemical) exposure variables were z-score standardized. All models were adjusted for age, sex, BMI, residual WC, physical activity and smoking, educational attainment, prevalent hypertension, prevalent diabetes, prevalent cancer (except for nonmelanoma skin cancer), prevalent cardiovascular disease, and antihypertensive medication. The lifestyle-associated variables were mutually adjusted for the remaining factors, that is, alcohol consumption, health satisfaction, and sleeping duration. The food intake variables were additionally energy-adjusted (per $1000 \mathrm{kcal}$ ).
In sensitivity analyses, individuals with the following characteristics were excluded from the analyses or evaluated separately: women $(n=1494)$, prevalent cancer (except nonmelanoma skin types), prevalent cardiovascular disease, or prevalent type 2 diabetes $(n=182)$, and hs-CRP of $10 \mathrm{mg} / \mathrm{L}$ or higher that could reflect acute inflammation $(n=78)$ (31). Differences in the associations according to sex were tested on the multiplicative scale based on calculated interaction terms of the respective variables and sex variable. All statistical analyses were performed in SAS (version 9.4, Enterprise Guide 7.1, SAS Institute Inc., Cary, NC, USA) and R (version 3.4.3, R Foundation for Statistical Computing, Vienna, Austria).

\section{Results}

Table 1 presents the descriptive characteristics of the study population, overall and stratified by sex. The median (IQR) of chemerin concentration was $147.6(125.6,172.4) \mathrm{ng} / \mathrm{mL}$. The participants had a mean (S.D.) age of 50.3 (9.0) years, and their average BMI was $26.1(4.3) \mathrm{kg} / \mathrm{m}^{2}$. Overall, men were more likely to be smokers and showed higher alcohol consumption. The distribution of evaluated lifestyleassociated, dietary, and metabolic factors according to quartiles of chemerin concentrations are presented in Supplementary Table 3. These analyses, among others, revealed a trend of increasing chemerin concentrations with reduced wine consumption, longer sleep duration, higher prevalence of sleeping disorders, level of health dissatisfaction and physical inactivity.

Figure 2 shows the results from RFR depicting the importance of evaluated variables as statistical predictors of chemerin concentrations among the three groups of factors, that is, lifestyle-associated, dietary, and metabolic, as well as all predictors modeled together. Among the lifestyle-associated factors, BMI and WC were selected as the most important predictors of circulating chemerin, followed by sleeping duration and alcohol intake. Among the dietary factors, consumption of red meat and processed meat, fruits, SSB, vegetables, dairy products, and refined grains were selected as main predictors of chemerin concentrations. With regard to metabolic factors, FLI, hs-CRP, and eGFR, followed by triglycerides and creatinine showed highest importance in the prediction of chemerin. In an analysis that evaluated the relative variable importance of all predictors together, hs-CRP was the most important predictor of circulating chemerin, followed by FLI, eGFR, BMI, WC, and triglycerides.

In linear regression analyses, the set of variables selected by RFR explained 32.9\% variation of chemerin

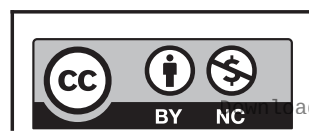

This work is licensed under a Creative Commons Attribution-NonCommercial 4.0 International License. ded from Bioscientifica.com at 04/26/2023 09:43:50AM 
concentrations (lifestyle-associated factors including BMI, WC, sports, smoking, alcohol, sleeping duration, and health satisfaction: 18.1\%; dietary factors including processed meat, red meat, fish, dairy, eggs, legumes, vegetables, fruits, nuts, whole grains, refined grains, SSB: 1.7\%; metabolic factors including hs-CRP, total cholesterol, LDL-C, HDLC, triglycerides, FLI, ALT, GGT, uric acid, creatinine, eGFR, systolic BP, diiastolic BP: 27.7\%) (Supplementary Table 4). Overall, the full range of evaluated variables jointly explained $35.4 \%$ of chemerin concentrations.

Figure 3 shows results from the multivariable-linear regressionanalyses that furtherdemonstratethedirection and strength of the associations between chemerin and selected determinants. For example, higher chemerin concentrations were associated with higher BMI (standardized beta $=2.9$ $(95 \%$ CI: $2.6,3.2) \mathrm{ng} / \mathrm{mL})$ and WC $(1.2$ (95\% CI: 1.0, 1.3) ng/ $\mathrm{mL})$, longer sleeping duration (1.3 (95\% CI: $0.2,2.5) \mathrm{ng} / \mathrm{mL})$, higher intakes of red meat and SSB (1.7 (95\% CI: $0.5,3.0) \mathrm{ng} /$ $\mathrm{mL}$ and 1.7 (95\% CI: 0.4, 2.9) ng/mL, respectively), and lower intakes of dairy (-1.5 (95\% CI: $-2.8,-0.2) \mathrm{ng} / \mathrm{mL})$.

In analyses stratified by sex, WC, red meat, dairy, FLI, and hs-CRP were selected as main statistical predictors in RFR analysis for both men and women (Fig. 4). Relative to WC, BMI showed high importance in women and less in men. Sleeping duration and intakes of eggs, fruits, and vegetables were further selected as important predictors in the analyses for women, whereas smoking and intake of nuts were selected as important predictors in the analyses for men. Multivariable-adjusted linear regression analyses stratified by sex additionally characterized the suggested differences (Supplementary Table 5). For example, red meat consumption was associated with increasing chemerin concentrations in women only (standardized beta $=1.8$ (95\% CI: 0.1, 3.5) $\mathrm{ng} / \mathrm{mL}$ ), and the association with FLI was only present in men (3.0 (95\% CI: $0.3,5.6) \mathrm{ng} / \mathrm{mL}$ ). However, no statistically significant interaction by sex could be seen for the majority of the factors, with the exception of red meat consumption $\left(P_{\text {interaction }}=0.02\right)$.

In sensitivity analyses, the observed associations were not substantially altered after excluding participants with any prevalent disease. After excluding participants with elevated hs-CRP ( $\geq 10 \mathrm{mg} / \mathrm{L}$ ), the associations with SSB and FLI were attenuated toward null (Supplementary Table 6).

\section{Discussion}

In this large population-based study, we explored potential determinants of elevated chemerin concentrations among a wide range of lifestyle-associated, dietary, and metabolic factors in adult asymptomatic individuals. Our analyses highlighted the importance of BMI, WC, hs-CRP, FLI, and eGFR as main statistical predictors of chemerin concentrations. Systolic and diastolic blood pressure and triglycerides also contributed to the variation of circulating chemerin. Modifiable dietary and lifestyle-associated factors were further suggested to predict chemerin concentrations, albeit to a lesser degree. These included intakes of red and processed meat, SSB, dairy, and sleeping duration. Factors that were associated with increased concentrations of chemerin included physical inactivity and health dissatisfaction. Additional predictors of elevated chemerin concentrations included alcohol consumption and intakes of refined grains, fruits, and vegetables.

This is the first observational study to explore such a wide range of factors covering various phenotypes, including modifiable lifestyle and dietary factors in relation to elevated chemerin concentrations in predominantly healthy adults. Yet, our results notably distinguished inflammatory and metabolic factors as the most important determinants of elevated chemerin concentrations. So far, various lines of research characterized chemerin as a potential player in the development of cardio-metabolic diseases and described its multifaceted functions in the regulation of energy metabolism, adipogenesis, and angiogenesis (32). Systemically elevated chemerin could originate from various sources representing tissue damage or immune activation in different organs with regulatory actions in various inflammatory processes (32). Thus, it may not be surprising that its elevated concentrations coincide with elevated levels of other inflammation markers such as CRP as shown in our data. However, CRP is a non-specific biomarker of inflammation and may also reflect obesity-associated inflammatory phenotype. Indeed, the enlarged adipose tissue is one important source of secretion of pro-inflammatory mediators (33). Chemerin can be also characterized as one of those mediators, as it is predominantly expressed in adipocytes within white adipose tissue, and its association with obesity is well established (4). Chemerin is expressed similarly in human preadipocytes and adipocytes but can also be found in the stroma-vascular fraction, suggesting that the different adipose tissue cell types may contribute to chemerin production (34). In addition, immune cells such as macrophages, dendritic cell subsets, and natural killer cells express CMKLR1 and are chemerin responsive (2). Furthermore, higher chemerin concentrations were associated with larger amounts of visceral adipose tissue (VAT) as compared to lower amounts of subcutaneous adipose tissue (SAT) $(4,35)$. Interestingly, our data revealed

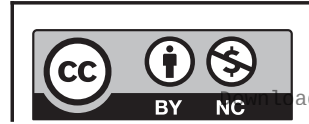

This work is licensed under a Creative Commons Attribution-NonCommercial 4.0 International License. ded from Bioscientifica.com at 04/26/2023 09:43:50AM 
Table 1 Descriptive characteristics of the study population, overall and by sex. Dietary intake and clinical parameters are expressed as median (IQR).

\begin{tabular}{|c|c|c|c|c|}
\hline & & Total, $n=2433$ & Men, $n=939$ & Women, $n=1494$ \\
\hline & Chemerin (ng/mL), median (IQR) & $147.6(125.6,172.4)$ & $145.6(126.5,170.2)$ & $147.6(125.6,172.1)$ \\
\hline \multicolumn{5}{|l|}{ Socio-demographic factors } \\
\hline & Age (years), mean (s.D.) & $50.3(9.0)$ & $52.4(8.1)$ & $49.0(9.3)$ \\
\hline Education & University degree, $n(\%)$ & $935(38.4)$ & $496(52.8)$ & $439(29.4)$ \\
\hline \multicolumn{5}{|l|}{ Health status } \\
\hline \multirow[t]{2}{*}{ Prevalent condition } & $\begin{array}{l}\text { Cardiovascular disease and/or } \\
\text { hypertension, } n(\%)\end{array}$ & $1270(52.2)$ & $624(66.5)$ & $646(43.2)$ \\
\hline & Type 2 diabetes and/or cancer, $n(\%)$ & $249(10.3)$ & $100(10.7)$ & $149(10)$ \\
\hline \multirow[t]{3}{*}{ Medication use } & $\begin{array}{l}\text { Antihypertensive, anti-coagulant, } \\
\text { lipid-lowering, } n(\%)\end{array}$ & $632(26.0)$ & $291(31.0)$ & $341(22.9)$ \\
\hline & Aspirin, $n(\%)$ & $246(10.1)$ & $89(9.5)$ & $157(10.5)$ \\
\hline & Anti-diabetic, $n(\%)$ & $58(2.4)$ & $36(3.8)$ & $22(1.5)$ \\
\hline \multicolumn{5}{|l|}{ Lifestyle-associated factors } \\
\hline \multirow[t]{2}{*}{ Obesity measures } & Body mass index $\left(\mathrm{kg} / \mathrm{m}^{2}\right)$, mean (s.D.) & $26.1(4.3)$ & $26.8(3.5)$ & $25.7(4.6)$ \\
\hline & Waist circumference (cm), mean (s.D.) & $85.8(12.8)$ & $94.2(9.9)$ & $80.6(11.6)$ \\
\hline \multirow[t]{2}{*}{ Physical activity } & $\begin{array}{l}\text { Recreational sports (h/week), median } \\
\text { (IQR) }\end{array}$ & $4.5(2.0,8.0)$ & $5.5(2.5,9.5)$ & $4.0(2.0,7.5)$ \\
\hline & Inactive, $n(\%)$ & $596(24.7)$ & $220(23.5)$ & $376(25.4)$ \\
\hline Smoking & Ever smoker, $n(\%)$ & $1290(53.0)$ & $665(70.8)$ & $625(41.8)$ \\
\hline \multirow[t]{4}{*}{ Alcohol consumption } & $\begin{array}{l}\text { Intake of consumers (g/day), } \\
\text { median (IQR) }\end{array}$ & $8.5(3.1,20.1)$ & $18.4(8.6,32.5)$ & $5.2(2.1,10.6)$ \\
\hline & Non-consumers, $n(\%)$ & $72(3.0)$ & $32(3.4)$ & $40(2.7)$ \\
\hline & $\begin{array}{l}\text { Light and moderate drinkers (<15 } \\
\text { g/day women; <30 g/day men), } n(\%)\end{array}$ & $1859(76.5)$ & $665(70.8)$ & $1194(80.0)$ \\
\hline & $\begin{array}{l}\text { Heavy drinkers ( } \geq 15 \text { g/day women; } \\
\geq 30 \text { g/day men), } n(\%)\end{array}$ & $502(20.6)$ & $242(25.8)$ & $260(17.4)$ \\
\hline \multirow[t]{2}{*}{ Sleeping duration } & Short (<7 h/day), $n(\%)$ & $609(25.0)$ & $249(26.5)$ & $360(24.1)$ \\
\hline & Long (>8 h/day), $n$ (\%) & $303(12.5)$ & $114(12.1)$ & $189(12.6)$ \\
\hline Self-reported well-being & $\begin{array}{l}\text { Health satisfaction - Dissatisfied, } \\
n(\%)\end{array}$ & $432(17.8)$ & $141(15.0)$ & $291(19.5)$ \\
\hline \multicolumn{5}{|l|}{ Dietary factors } \\
\hline & Processed meat (g/day) & $49.8(31.6,76.3)$ & $63.4(45.4,101.3)$ & $43.0(25.1,59.9)$ \\
\hline & Red meat (g/day) & $36.5(23.3,54.7)$ & $47.7(32.5,69.8)$ & $31.7(19.3,45.5)$ \\
\hline & Fish (g/day) & $18.4(9.9,29.0)$ & $23.0(11.2,34.8)$ & $16.4(9.0,28.5)$ \\
\hline & Dairy (g/day) & $170.9(94.5,276.7)$ & $148.8(73.6,251.8)$ & $191.7(106.7,310.5)$ \\
\hline & Eggs (g/day) & $13.6(9.0,21.9)$ & $16.0(9.0,25.3)$ & $13.6(7.8,20.7)$ \\
\hline & Legumes (g/day) & $16.5(9.1,30.3)$ & $21.6(11.0,38.6)$ & $13.9(8.0,26.3)$ \\
\hline & Vegetables (g/day) & $93.1(66.5,127.1)$ & $83.1(59.4,114.4)$ & $98.9(71.1,134.9)$ \\
\hline & Fruits (g/day) & $122.4(91.5,202.3)$ & $107.9(82.0,180.9)$ & $140.5(96.4,215.6)$ \\
\hline & Nuts (g/day) & $0.8(0.4,4.1)$ & $0.8(0.4,4.1)$ & $0.8(0.4,4.1)$ \\
\hline & Whole grains (g/day) & $32.0(8.7,75.1)$ & $22.1(5.5,63.0)$ & $37.4(11.3,82.0)$ \\
\hline & Refined grains (g/day) & $134.1(86.3,190.5)$ & $175.9(119.8,231.7)$ & $112.4(72.5,160.5)$ \\
\hline & Sugar-sweetened beverages (g/day) & $1.4(0.0,24.7)$ & $5.6(0.0,48.3)$ & $0.0(0.0,9.7)$ \\
\hline \multicolumn{5}{|l|}{ Metabolic factors } \\
\hline Chronic inflammation & hs-CRP ( $\mu \mathrm{g} / \mathrm{mL})$ & $0.8(0.2,2.2)$ & $0.7(0.2,1.8)$ & $0.9(0.2,2.6)$ \\
\hline \multirow[t]{4}{*}{ Lipid metabolism } & Total cholesterol (mmol/L) & $5.3(4.6,6.0)$ & $5.4(4.8,6.1)$ & $5.1(4.5,5.9)$ \\
\hline & LDL-C (mmol/L) & $3.1(2.5,3.7)$ & $3.2(2.7,3.8)$ & $3.0(2.4,3.6)$ \\
\hline & $\mathrm{HDL}-\mathrm{C}(\mathrm{mmol} / \mathrm{L})$ & $1.4(1.2,1.7)$ & $1.3(1.1,1.5)$ & $1.5(1.3,1.8)$ \\
\hline & Triglycerides (mmol/L) & $1.2(0.9,1.8)$ & $1.6(1.1,2.3)$ & $1.1(0.8,1.5)$ \\
\hline \multirow[t]{2}{*}{ Liver function } & FLI & $0.5(0.1,2.1)$ & $1.5(0.5,4.1)$ & $0.2(0.1,0.8)$ \\
\hline & $\mathrm{ALT}(\mathrm{U} / \mathrm{L})$ & $20.0(15.0,28.0)$ & $27.0(20.0,38.0)$ & $17.0(14.0,22.0)$ \\
\hline \multirow[t]{4}{*}{ Kidney function } & GGT (U/L) & $18.0(12.0,33.0)$ & $28.0(18.0,48.0)$ & $14.0(9.0,22.0)$ \\
\hline & Uric acid (mg/dL) & $4.5(3.7,5.5)$ & $5.6(4.8,6.3)$ & $4.1(3.4,4.8)$ \\
\hline & Creatinine (mg/dL) & $0.8(0.7,1.0)$ & $1.0(0.9,1.1)$ & $0.8(0.7,0.8)$ \\
\hline & eGFR (mL/min per $\left.1.73 \mathrm{~m}^{2}\right)$ & $91.1(79.7,101.0)$ & $91.0(80.4,99.8)$ & $91.1(79.5,101.7)$ \\
\hline \multirow[t]{2}{*}{ Blood pressure } & Systolic (mmHg), mean (s.D.) & $129.3(17.6)$ & $135.3(17.1)$ & $125.6(16.8)$ \\
\hline & Diastolic (mmHg), mean (s.D.) & $83.7(10.6)$ & $86.8(10.7)$ & $81.7(10.0)$ \\
\hline
\end{tabular}

ALT, alanine aminotransferase; BP, blood pressure; CRP, C-reactive protein; eGFR, estimated glomerular filtration rate; FLI, fatty liver index; g, grams; GGT, gamma-glutamyl transferase; h, hour; hs, high-sensitivity; IQR, interquartile range.

https://ec.bioscientifica.com https://doi.org/10.1530/EC-21-0273 (c) 2021 The authors Published by Bioscientifica Lto

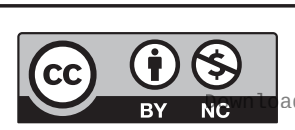

This work is licensed under a Creative Commons Attribution-NonCommercial 4.0 International License. Aed from Bioscientifica com at 04/26/2023 09:43:50Am via free access 
WC as a strong lifestyle-associated predictor of elevated chemerin concentrations in men and women, whereas BMI was shown as a strong predictor in women but not in men. WC is a measure of body fat distribution that is suggested to reflect visceral adiposity. In contrast, BMI is a measure of body composition and does not distinguish body fat and lean muscle mass (36). It is well known that as they age men and women are characterized by differing fat distribution profiles, such that men tend to accumulate fat in VAT whereas in women fat is stored predominantly in SAT depots. VAT is metabolically more active and may better reflect early inflammation-related pathological conditions as compared to SAT. In this context, our data may provide an important insight that chemerin could serve as a biomarker that can depict VAT-associated inflammatory phenotypes in both sexes. Although WC is often used as a proxy measure of VAT and has shown to be stronger correlated to VAT than BMI (37), it may still not be the best method to estimate visceral fat accumulation (38). Further studies that employ precise assessment of fat compartments would be needed.

Besides adipose tissue, chemerin is also expressed in the liver (32) and kidney (39). In our study, FLI and eGFR were among the most important metabolic predictors of chemerin, followed by triglycerides and creatinine. Also systolic and diastolic blood pressure were selected to contribute to the variation of chemerin. Similar results were previously reported for an association between chemerin and impaired renal function $(39,40)$ and other metabolic phenotypes that may lead to liver and kidney damage (4, 41,42 ). Our findings for blood pressure are supported by mechanistic research on chemerin that has been focused on the vascular system and hypertension (43). Among these, human and animal studies revealed that chemerin
A

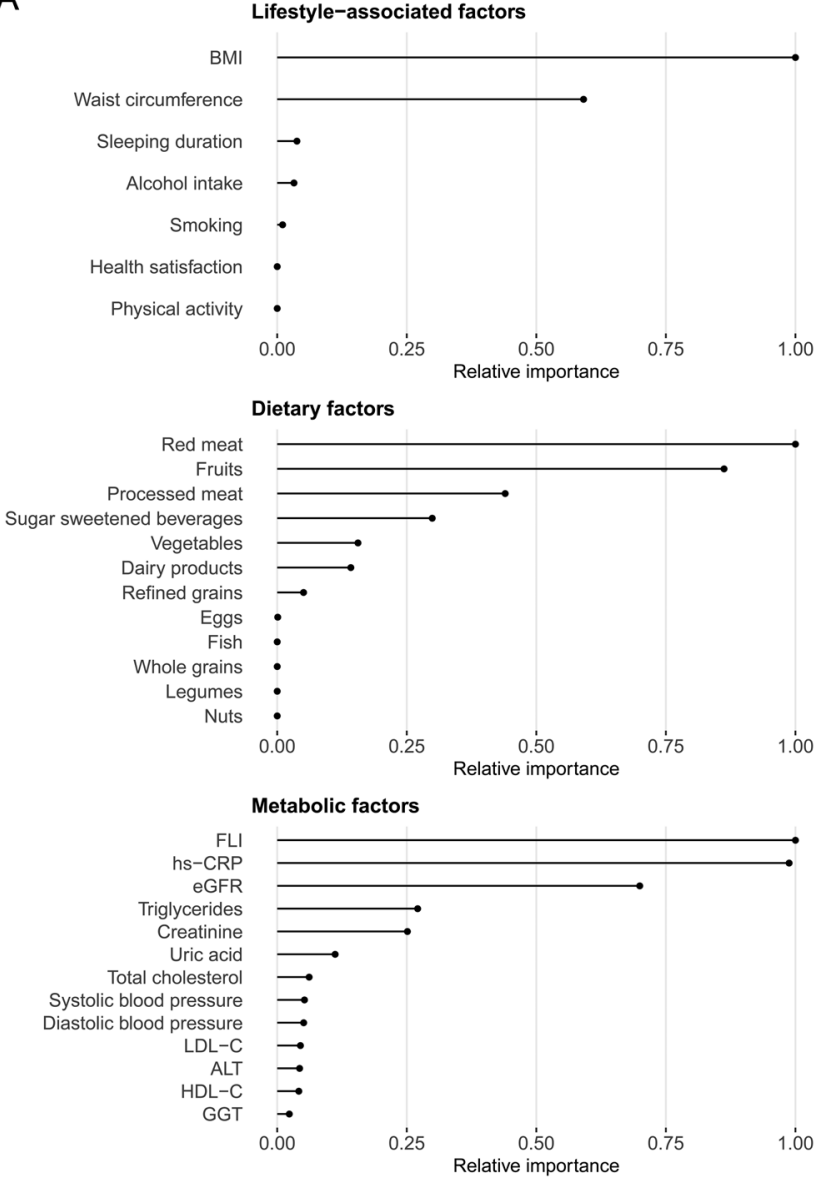

B

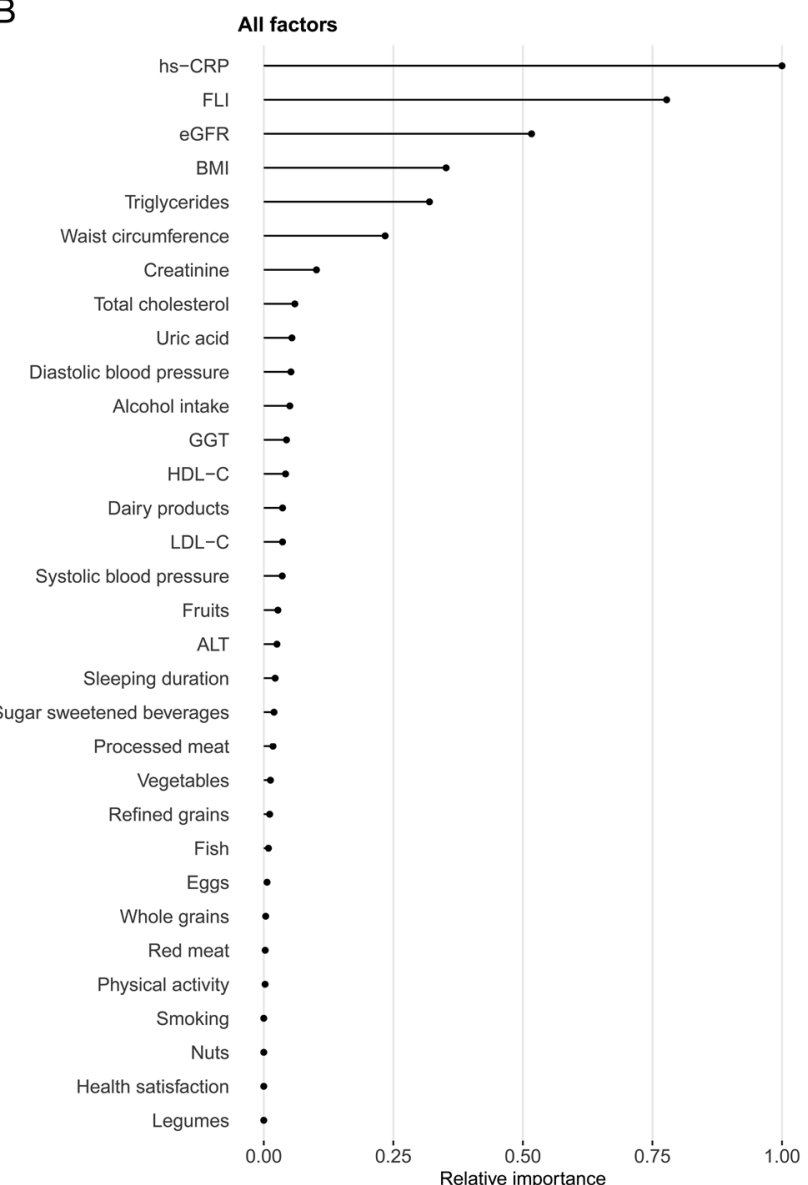

Figure 2

Relative variable importance from random forest regression for lifestyle-associated, dietary, and metabolic factors as predictors of chemerin concentrations (A) modeled separately per block and (B) modeled together. The potential predictors are plotted relative to the most important predictor of circulating chemerin by (A) block of lifestyle-associated, dietary, and metabolic factors and (B) all factors together. ALT, alanine aminotransferase; BP, blood pressure; eGFR, estimated glomerular filtration rate; FLI, fatty liver index; GGT, gamma-glutamyl transferase.

https://ec.bioscientifica.com https://doi.org/10.1530/EC-21-0273 (c) 2021 The authors Published by Bioscientifica Ltd
This work is licensed under a Creative Commons Attribution-NonCommercial 4.0 International License. ded from Bioscientifica.com at $04 / 26 / 2023$ 09:43:50AM 


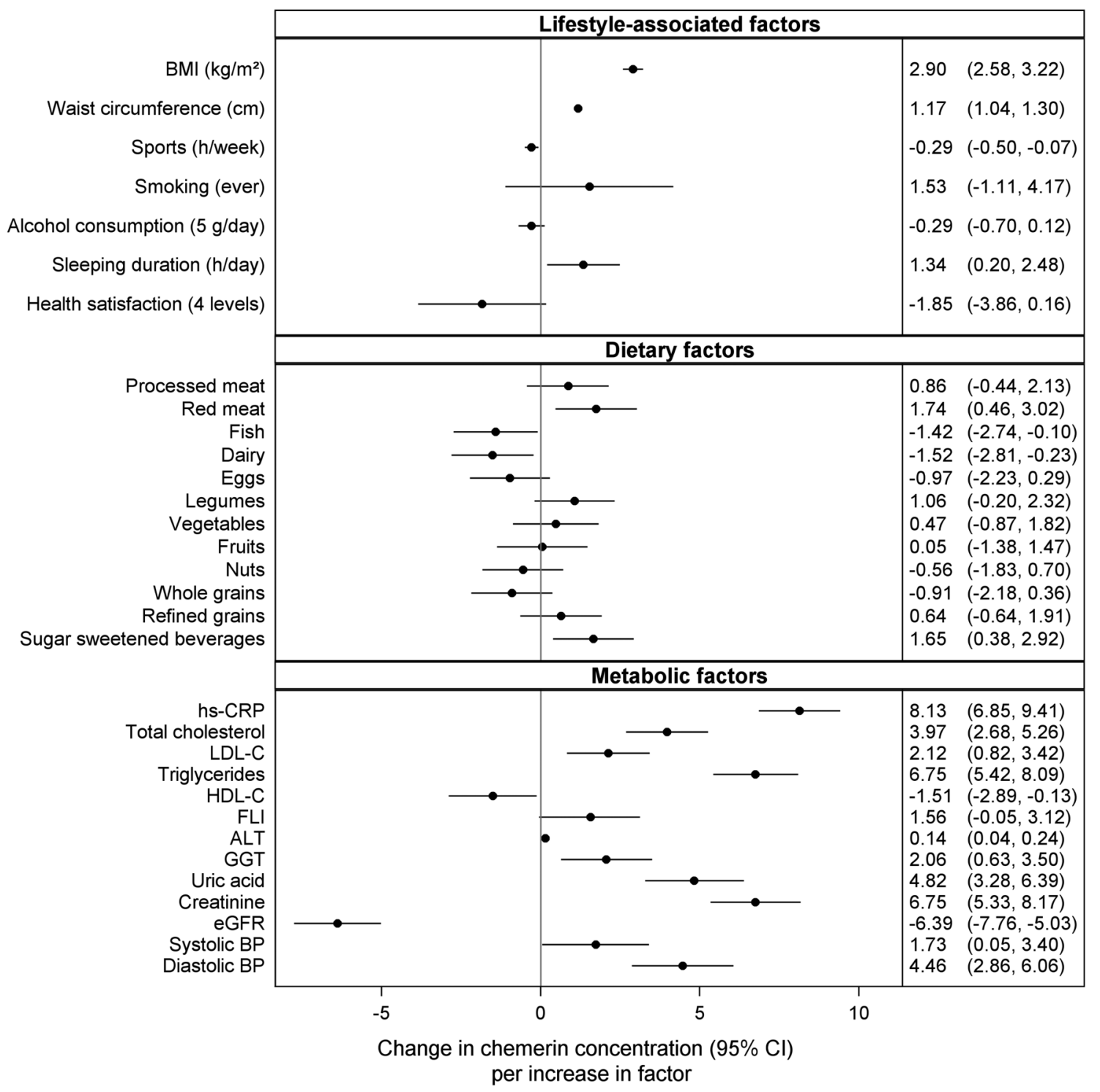

Figure 3

Multivariable linear regression showing change in chemerin concentration per increase in lifestyle-associated, dietary, and metabolic factors. Multivariableadjusted model includes age, sex, BMI, residual waist circumference, physical activity, educational attainment, smoking status, prevalent hypertension, prevalent diabetes, prevalent cancer, prevalent cardivascular disease, and antihypertensive medication. The lifestyle-associated factors were mutually adjusted for the remaining factors, that is, additionally adjusted for alcohol consumption, health satisfaction, and sleeping duration. Dietary factors and metabolic factors were not mutually adjusted for one another. Sleeping duration was additionally adjusted for sleeping disorders and consumption of caffeinated beverages (tea and coffee). Diet was z-score standardized and energy-adjusted; metabolic exposure variables were z-score standardized. ALT, alanine aminotransferase; BP, blood pressure; eGFR, estimated glomerular filtration rate; FLI, fatty liver index; GGT, gamma-glutamyl transferase.

causes contraction of arteries and increases reactive oxygen species (ROS) in endothelial cells (43). Overall, our data come in support of previous research that implicates chemerin as a biomarker of immunometabolism, linking obesity, inflammation, and metabolic disorders.

In addition to metabolic determinants, our results revealed a number of lifestyle-associated factors associated with elevated chemerin concentrations. Among these, sleeping duration and alcohol consumption were rated among the most important predictors. Our results for an association between long sleep duration (sleep $>8 \mathrm{~h} /$ day) and chemerin independent of potential confounders such as prevalent chronic diseases or age are in line with findings from a recent meta-analysis, where long sleeping hours were associated with higher levels of pro-inflammatory biomarkers, i.e., CRP and IL-6 (44). Recent evidence from https://ec.bioscientifica.com https://doi.org/10.1530/EC-21-0273 (c) 2021 The authors Published by Bioscientifica Ltd

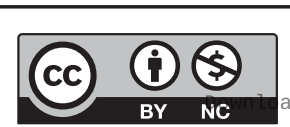

This work is licensed under a Creative Commons Attribution-NonCommercial 4.0 International License. ded from Bioscientifica.com at 04/26/2023 09:43:50AM via free access 
A
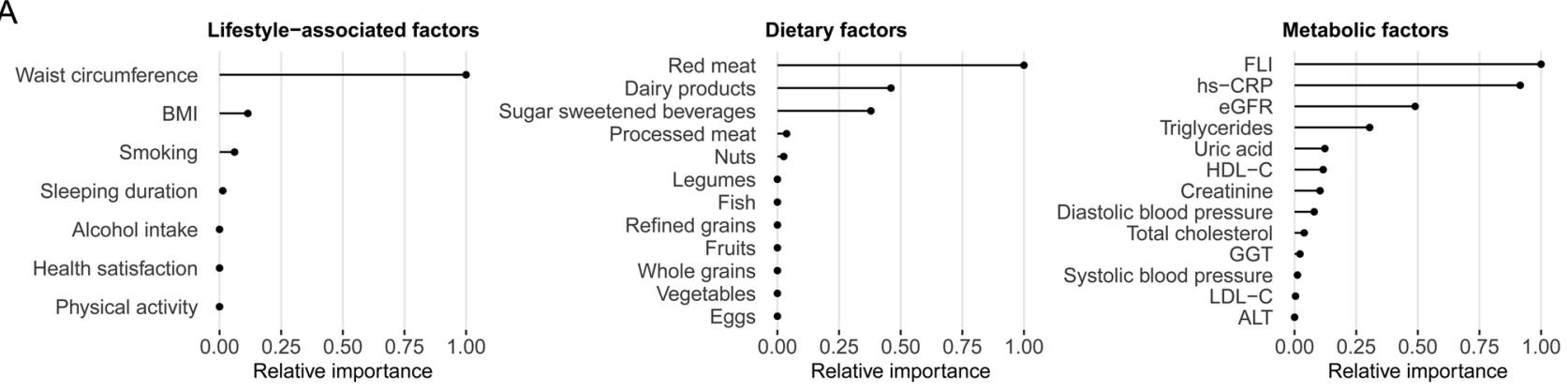

B
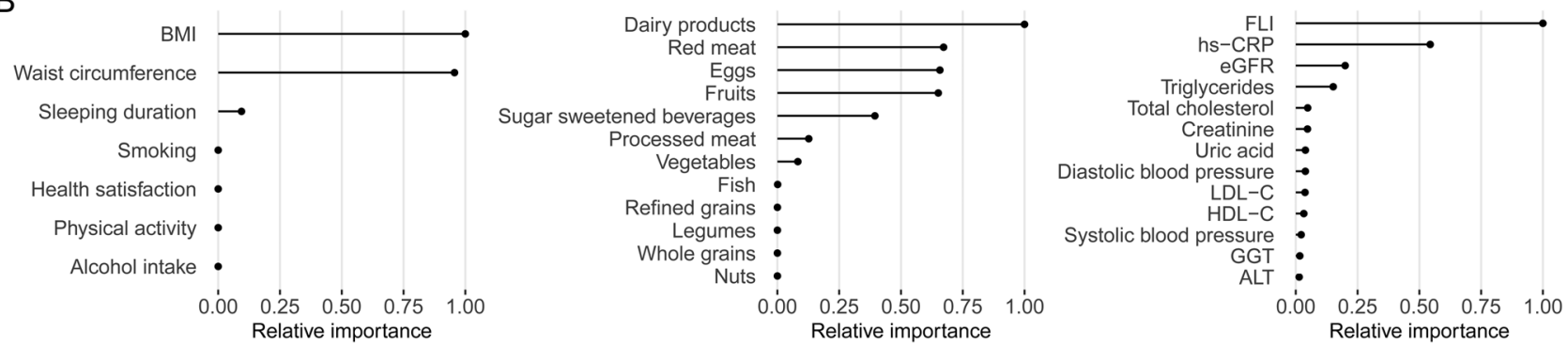

\section{Figure 4}

Relative variable importance from random forest regression for lifestyle-associated, dietary, and metabolic factors as predictors of chemerin concentrations in (A) men and (B) women. The potential predictors are plotted relative to the most important predictor of circulating chemerin per block (lifestyle-associated, dietary, and metabolic factors). ALT, alanine aminotransferase; BP, blood pressure; eGFR, estimated glomerular filtration rate; FLI, fatty liver index; GGT, gamma-glutamyl transferase.

prospective studies propose that the relationship between hours of sleep and risk of coronary events or mortality is U-shaped (45). Future prospective studies that account for additional potential confounders such as stress levels may be needed to better evaluate the association between sleep and chemerin levels. We also observed independent associations of chemerin with physical inactivity and health dissatisfaction. Not only is visceral fat accumulation a consequence of inactivity, but accumulating evidence suggests that exercise has anti-inflammatory effects independent of obesity status (46). One hypothesis is that exercise can downregulate the expression of TLR4 and reduce activation of NLRP3 inflammasome, reducing cytokine levels (46). The association with health dissatisfaction could be explained by the influence of prevalent disease status, mental health, and chronic stress, as inflammation is induced by these stressors (47). Overall, our data largely point to the importance of accounting for the complexity of chemerin determinants following a holistic approach that covers not just biological and physiological domains but also behavioral, emotional, and social well-being aspects.

Our data further revealed that higher alcohol consumption - and wine in particular - was associated with lower chemerin concentrations. This finding is in contrast to some previous studies which reported that alcohol was positively associated with chemerin levels (13). However, those studies did not differentiate between type of alcoholic drink and did not adjust for other potential confounders such as dietary and metabolic factors. The hypothesis that moderate wine consumption may favorably influence inflammatory status is strengthened by experimental research showing beneficial properties of polyphenols abundant in wine in the regulation of chemerin expression $(48,49)$. Furthermore, wine consumption is typical for the Mediterranean-style diet as one of the most commonly reported dietary patterns in relation to lower inflammation levels (50). Further randomized control trials are warranted to evaluate the suggested link between wine consumption in modulating chemerin concentrations.

Among the dietary factors, our data suggested red and processed meat, fruits, SSB, vegetables, dairy products, and refined grains as potentially important determinants of elevated chemerin concentrations. Linear associations with high intakes of SSB and red meat and low intakes of dairy products with elevated circulating chemerin were observed. These findings come in support of the increasing evidence revealing the pro-inflammatory potential of consuming Western diets $(51,52,53)$ vs the anti-inflammatory properties of food components of the Mediterranean diet (54). For example, dairy has been studied as part of high protein diet in previous work, and results

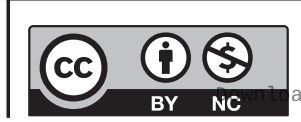

This work is licensed under a Creative Commons Attribution-NonCommercial 4.0 International License. ded from Bioscientifica.com at 04/26/2023 09:43:50AM 
revealed decreasing effects on chemerin levels following dietary interventions $(55,56)$. The anti-inflammatory effects of dairy could be accounted for by various reasons, including benefits of specific fatty acids in dairy fat (such as branch-chain fatty acids, medium-chain saturated fats, specific trans fats), benefits of fermentation that may interact with microbiome, benefits of probiotics, or other unknown bioactivities (57). The importance of fruits comes in line with studies suggesting high intakes of fruits lead to a reduction in pro-inflammatory mediators and enhanced immune cell profile (58). The association, however, was not well captured in our linear regression model and seemed rather U-shaped, deserving attention in future research. The inverse associations found with fish and whole grains in women also fit to the hypothesis of protective effects of a Mediterranean-type diet; however, these variables were not selected as important predictors from RFR analyses.

Our findings suggested differences in variable importance by sex, that is, sleeping duration, smoking, and intake of eggs, fruits, vegetables, and nuts. Various physiological and psychosocial differences may have possibly accounted for these differences. For instance, women are more often exposed to stressors that may have inflammatory consequences (59). Having the reduced statistical power in these stratified analyses, further studies with larger samples are warranted to explore sex-specific associations with elevated chemerin concentrations.

We must acknowledge several limitations of our study: (1) the cross-sectional study design limits interpretation on temporal links between evaluated determinants and chemerin concentrations; (2) the measurement of dietary intakes is error prone and less precise than biomarker measurements. Assuming that measurement error was independent of chemerin concentrations or related factors, this would bias any potential association toward the null. Associations of chemerin concentrations with dietary factors could be underestimated; (3) chemerin concentrations may show day/night variations (59) which may influence observed associations with various traits. However, in our study, biosample collections were taken only over daytime, minimizing the potential influence of circadian variation; (4) residual confounding from subclinical disease could not be excluded in our adjusted models.

\section{Conclusions}

In this large population-based study, we explored potential determinants of elevated chemerin concentrations among a wide range of lifestyle-associated, dietary, and metabolic factors. The findings come in support of the role of chemerin characterizing inflammatory and metabolic phenotypes in predominantly healthy adults. Modifiable dietary and lifestyle-associated determinants of elevated chemerin concentrations require further evaluation in a prospective study setting.

\section{Supplementary materials}

This is linked to the online version of the paper at https://doi.org/10.1530/ EC-21-0273.

\section{Declaration of interest}

The authors declare that there is no conflict of interest that could be perceived as prejudicing the impartiality of the research reported.

\section{Funding}

The study was supported by the Federal Ministry of Science, Germany (grant 01 EA 9401) and the European Union (grant SOC 9520140805 F02) for the recruitment phase of the EPIC-Potsdam study and by the German Cancer Aid (grant 70-2488-Ha I) and the European Community (grant SOC 9820076905 F02) for the follow-up of the EPIC-Potsdam study. This work was also partly supported by the German Research Foundation (DFG) (grant AL 1784/3-1) which has funded the research position of KA for the time of study conduct and analysis. The funders had no role in study design, data collection and analysis, decision to publish, or preparation of the manuscript.

\section{Acknowledgements}

The authors thank the Human Study Centre (HSC) of the German Institute of Human Nutrition Potsdam-Rehbrücke, the trustee and the data hub for the processing, the participants for the provision of the data, the biobank for the processing of the biological samples, and the head of the HSC, Manuela Bergmann, for the contribution to the study design and leading the underlying processes of data generation.

\section{References}

1 Helfer G \& Wu QF. Chemerin: a multifaceted adipokine involved in metabolic disorders. Journal of Endocrinology 2018238 R79-R94. (https://doi.org/10.1530/JOE-18-0174)

2 Zabel BA, Kwitniewski M, Banas M, Zabieglo K, Murzyn K \& Cichy J. Chemerin regulation and role in host defense. American Journal of Clinical and Experimental Immunology 20143 1-19.

3 Kaur J, Mattu HS, Chatha K \& Randeva HS. Chemerin in human cardiovascular disease. Vascular Pharmacology 2018110 1-6. (https:// doi.org/10.1016/j.vph.2018.06.018)

4 Zylla S, Pietzner M, Kühn JP, Völzke H, Dörr M, Nauck M \& Friedrich N. Serum chemerin is associated with inflammatory and metabolic parameters-results of a population-based study. Obesity 201725 468-475. (https://doi.org/10.1002/oby.21735)

5 Eichelmann F, Schulze MB, Wittenbecher C, Menzel J, Weikert C, Giuseppe Rd, Biemann R, Isermann B, Fritsche A, Boeing H, et al. Chemerin as a biomarker linking inflammation and cardiovascular diseases. Journal of the American College of Cardiology 201973 378-379. (https://doi.org/10.1016/j.jacc.2018.10.058) 
6 Zylla S, Dörr M, Völzke H, Schminke U, Felix SB, Nauck M \& Friedrich N. Association of circulating chemerin with subclinical parameters of atherosclerosis: results of a population-based study. Arteriosclerosis, Thrombosis, and Vascular Biology 201838 1656-1664. (https://doi.org/10.1161/ATVBAHA.118.311219)

7 Menzel J, di Giuseppe R, Biemann R, Wittenbecher C, Aleksandrova K, Eichelmann F, Fritsche A, Schulze MB, Boeing H, Isermann B, et al. Association between chemerin, omentin-1 and risk of heart failure in the population-based EPIC-potsdam study. Scientific Reports $2017 \mathbf{7}$ 14171. (https://doi.org/10.1038/s41598-017-14518-2)

8 Eichelmann F, Schulze MB, Wittenbecher C, Menzel J, Weikert C, di Giuseppe R, Biemann R, Isermann B, Fritsche A, Boeing H, et al. Association of chemerin plasma concentration with risk of colorectal cancer. JAMA Network Open 20192 e190896. (https://doi.org/10.1001/ jamanetworkopen.2019.0896)

9 Zhou X, Tao Y, Chen Y, Xu W, Qian Z \& Lu X. Serum chemerin as a novel prognostic indicator in chronic heart failure. Journal of the American Heart Association 20198 e012091. (https://doi.org/10.1161/ JAHA.119.012091)

10 Chakaroun R, Raschpichler M, Klöting N, Oberbach A, Flehmig G, Kern M, Schön MR, Shang E, Lohmann T, Dreßler M, et al. Effects of weight loss and exercise on chemerin serum concentrations and adipose tissue expression in human obesity. Metabolism: Clinical and Experimental 201261 706-714. (https://doi.org/10.1016/j. metabol.2011.10.008)

11 Kim SH, Lee SH, Ahn KY, Lee DH, Suh YJ, Cho SG, Choi YJ, Lee DH, Lee SY, Hong SB, et al. Effect of lifestyle modification on serum chemerin concentration and its association with insulin sensitivity in overweight and obese adults with type 2 diabetes. Clinical Endocrinology 201480 825-833. (https://doi.org/10.1111/cen.12249)

12 Blüher M, Rudich A, Klöting N, Golan R, Henkin Y, Rubin E, Schwarzfuchs D, Gepner Y, Stampfer MJ, Fiedler M, et al. Two patterns of adipokine and other biomarker dynamics in a long-term weight loss intervention. Diabetes Care 201235 342-349. (https://doi.org/10.2337/ dc11-1267)

13 Lemming EW, Byberg L, Stattin K, Ahmad S, Lind L, Elmståhl S, Larsson SC, Wolk A \& Michaëlsson K. Dietary pattern specific protein biomarkers for cardiovascular disease: a cross sectional study in 2 independent cohorts. Journal of the American Heart Association 20198 e011860. (https://doi.org/10.1161/JAHA.118.011860)

14 Neuparth MJ, Proença JB, Santos-Silva A \& Coimbra S. The positive effect of moderate walking exercise on chemerin levels in Portuguese patients with type 2 diabetes mellitus. Journal of Investigative Medicine 201462 350-353. (https://doi.org/10.2310/JIM.0000000000000025)

15 Salha T, Andrijević D, Vrselja Z, Šerić V, Radić R \& Curic G. Chemerin blood levels are associated with MRI measured volumes of abdominal adipose tissue compartments and lifestyle choices. Acta Clinica Croatica 201756 663-672. (https://doi.org/10.20471/acc.2017.56.04.13)

16 Boeing H, Wahrendorf J \& Becker N. EPIC-Germany: a source for studies into diet and risk of chronic diseases: European investigation into cancer and nutrition. Annals of Nutrition and Metabolism 199943 195-204. (https://doi.org/10.1159/000012786)

17 Boeing H, Korfmann A \& Bergmann MM. Recruitment procedures of EPIC-Germany: European investigation into cancer and nutrition. Annals of Nutrition and Metabolism 199943 205-215. (https://doi. org/10.1159/000012787)

18 Bohlscheid-Thomas S, Hoting I, Boeing H \& Wahrendorf J. Reproducibility and relative validity of energy and macronutrient intake of a food frequency questionnaire developed for the German part of the EPIC project: European prospective investigation into cancer and nutrition. International Journal of Epidemiology 199726 (Supplement 1) S71-S81. (https://doi.org/10.1093/ije/26.suppl_1.s71)

19 Bechthold A, Boeing H, Schwedhelm C, Hoffmann G, Knuppel S, Iqbal K, De Henauw S, Michels N, Devleesschauwer B, Schlesinger S, et al. Food groups and risk of coronary heart disease, stroke and heart failure: a systematic review and dose-response meta-analysis of prospective studies. Critical Reviews in Food Science and Nutrition 2017 59 1071-1090. (https://doi.org/10.1080/10408398.2017.1392288)

20 Eichelmann F, Weikert C, di Giuseppe R, Biemann R, Isermann B, Schulze MB, Boeing H \& Aleksandrova K. Methodological utility of chemerin as a novel biomarker of immunity and metabolism. Endocrine Connections 20176 340-347. (https://doi.org/10.1530/EC-170098)

21 Enzenbach C, Kroger J, Zietemann V, Jansen EH, Fritsche A, Doring F, Boeing H \& Schulze MB. Erythrocyte membrane phospholipid polyunsaturated fatty acids are related to plasma C-reactive protein and adiponectin in middle-aged German women and men. European Journal of Nutrition 201150 625-636. (https://doi.org/10.1007/s00394011-0169-4)

22 Jacobs S, Kroger J, Floegel A, Boeing H, Drogan D, Pischon T, Fritsche A, Prehn C, Adamski J, Isermann B, et al. Evaluation of various biomarkers as potential mediators of the association between coffee consumption and incident type 2 diabetes in the EPIC-Potsdam Study. American Journal of Clinical Nutrition 2014100 891-900. (https://doi. org/10.3945/ajcn.113.080317)

23 Friedewald WT, Levy RI \& Fredrickson DS. Estimation of the concentration of low-density lipoprotein cholesterol in plasma, without use of the preparative ultracentrifuge. Clinical Chemistry 1972 18 499-502. (https://doi.org/10.1093/clinchem/18.6.499)

24 Levey AS, Stevens LA, Schmid CH, Zhang YL, Castro AF, Feldman HI, Kusek JW, Eggers P, Van Lente F, Greene T, et al. A new equation to estimate glomerular filtration rate. Annals of Internal Medicine 2009 150 604-612. (https://doi.org/10.7326/0003-4819-150-9-20090505000006)

25 Bedogni G, Bellentani S, Miglioli L, Masutti F, Passalacqua M, Castiglione A \& Tiribelli C. The fatty liver index: a simple and accurate predictor of hepatic steatosis in the general population. BMC Gastroenterology 20066 33. (https://doi.org/10.1186/1471230X-6-33)

26 Stekhoven DJ \& Bühlmann P. MissForest: non-parametric missing value imputation for mixed-type data. Bioinformatics 201228 112-118. (https://doi.org/10.1093/bioinformatics/btr597)

27 Cao Y, Willett WC, Rimm EB, Stampfer MJ \& Giovannucci EL. Light to moderate intake of alcohol, drinking patterns, and risk of cancer: results from two prospective US cohort studies. BMJ $20153 \mathbf{3 5 1}$ h4238. (https://doi.org/10.1136/bmj.h4238)

28 von Ruesten A, Weikert C, Fietze I \& Boeing H. Association of sleep duration with chronic diseases in the European prospective investigation into cancer and nutrition potsdam study. PLOS ONE 2012 7 e30972. (https://doi.org/10.1371/journal.pone.0030972)

29 Ryo M \& Rillig MC. Statistically reinforced machine learning for nonlinear patterns and variable interactions. Ecosphere 20178 e01976. (https://doi.org/10.1002/ecs2.1976)

30 Ishwaran H \& Kogalur UB. Fast unified random forests for survival, regression, and classification (RF-SRC), 2021. (available at: https://cran.r-project.org/web/packages/randomForestSRC/ randomForestSRC.pdf)

31 Black S, Kushner I \& Samols D. C-reactive protein. Journal of Biological Chemistry 2004279 48487-48490. (https://doi.org/10.1074/jbc. $\mathrm{R} 400025200)$

32 Ellulu MS, Patimah I, Khaza'ai H, Rahmat A \& Abed Y. Obesity and inflammation: the linking mechanism and the complications. Archives of Medical Science 201713 851-863. (https://doi.org/10.5114/ aoms.2016.58928)

33 Sell H, Laurencikiene J, Taube A, Eckardt K, Cramer A, Horrighs A, Arner P \& Eckel J. Chemerin is a novel adipocyte-derived factor inducing insulin resistance in primary human skeletal muscle cells. Diabetes 200958 2731-2740. (https://doi.org/10.2337/db09-0277)

34 Shin HY, Lee DC, Chu SH, Jeon JY, Lee MK, Im JA \& Lee JW. Chemerin levels are positively correlated with abdominal visceral fat accumulation. Clinical Endocrinology 201277 47-50. (https://doi. org/10.1111/j.1365-2265.2011.04217.x) 
35 Gurunathan U \& Myles PS. Limitations of body mass index as an obesity measure of perioperative risk. British Journal of Anaesthesia 2016 116 319-321. (https://doi.org/10.1093/bja/aev541)

36 Ross R, Neeland IJ, Yamashita S, Shai I, Seidell J, Magni P, Santos RD, Arsenault B, Cuevas A, Hu FB, et al. Waist circumference as a vital sign in clinical practice: a consensus statement from the IAS and ICCR Working Group on visceral obesity. Nature Reviews: Endocrinology 2020 16 177-189. (https://doi.org/10.1038/s41574-019-0310-7)

37 Brown JC, Harhay MO \& Harhay MN. The value of anthropometric measures in nutrition and metabolism: comment on anthropometrically predicted visceral adipose tissue and blood-based biomarkers: a cross-sectional analysis. Nutrition and Metabolic Insights 201912 1178638819831712. (https://doi org/10.1177/1178638819831712)

38 Pfau D, Bachmann A, Lössner U, Kratzsch J, Blüher M, Stumvoll M $\&$ Fasshauer M. Serum levels of the adipokine chemerin in relation to renal function. Diabetes Care 201033 171-173. (https://doi org/10.2337/dc09-1351)

39 Zylla S, Rettig R, Völzke H, Endlich K, Nauck M \& Friedrich N. Serum chemerin levels are inversely associated with renal function in a general population. Clinical Endocrinology 201888 146-153. (https:// doi.org/10.1111/cen.13449)

40 Bozaoglu K, Segal D, Shields KA, Cummings N, Curran JE, Comuzzie AG, Mahaney MC, Rainwater DL, VandeBerg JL, MacCluer JW, et al. Chemerin is associated with metabolic syndrome phenotypes in a Mexican-American population. Journal of Clinical Endocrinology and Metabolism 200994 3085-3088. (https://doi. org/10.1210/jc.2008-1833)

41 Chou HH, Teng MS, Hsu LA, Er LK, Wu S \& Ko YL. Circulating chemerin level is associated with metabolic, biochemical and haematological parameters: a population-based study. Clinical Endocrinology 202194 927-939. (https://doi.org/10.1111/cen.14441)

42 Ferland DJ, Mullick AE \& Watts SW. Chemerin as a driver of hypertension: a consideration. American Journal of Hypertension 2020 33 975-986. (https://doi.org/10.1093/ajh/hpaa084)

43 Irwin MR, Olmstead R \& Carroll JE. Sleep disturbance, sleep duration, and inflammation: a systematic review and meta-analysis of cohort studies and experimental sleep deprivation. Biological Psychiatry 2016 80 40-52. (https://doi.org/10.1016/j.biopsych.2015.05.014)

44 Cappuccio FP, D'Elia L, Strazzullo P \& Miller MA. Sleep duration and all-cause mortality: a systematic review and meta-analysis of prospective studies. Sleep 201033 585-592. (https://doi.org/10.1093/ sleep/33.5.585)

45 Burini RC, Anderson E, Durstine JL \& Carson JA. Inflammation, physical activity, and chronic disease: an evolutionary perspective sports medicine and health. Sports Medicine and Health Science 202059 1-6. (https://doi.org/10.1016/j.smhs.2020.03.004)

46 Liu YZ, Wang YX \& Jiang CL. Inflammation: the common pathway of stress-related diseases. Frontiers in Human Neuroscience 201711316. (https://doi.org/10.3389/fnhum.2017.00316)

47 Chalons P, Amor S, Courtaut F, Cantos-Villar E, Richard T, Auger C, Chabert P, Schni-Kerth V, Aires V \& Delmas D. Study of potential anti-inflammatory effects of red wine extract and resveratrol through a modulation of interleukin-1-beta in macrophages. Nutrients $2018 \mathbf{1 0}$ 1856. (https://doi.org/10.3390/nu10121856)

48 Kralisch S, Weise S, Sommer G, Lipfert J, Lossner U, Bluher M, Stumvoll M \& Fasshauer M. Interleukin-1beta induces the novel adipokine chemerin in adipocytes in vitro. Regulatory Peptides 2009 154 102-106. (https://doi.org/10.1016/j.regpep.2009.02.010)

49 Schwingshackl L \& Hoffmann G. Mediterranean dietary pattern, inflammation and endothelial function: a systematic review and meta-analysis of intervention trials. Nutrition, Metabolism, and Cardiovascular Diseases 201424 929-939. (https://doi.org/10.1016/j. numecd.2014.03.003)

50 Chai W, Morimoto Y, Cooney RV, Franke AA, Shvetsov YB, Le Marchand L, Haiman CA, Kolonel LN, Goodman MT \& Maskarinec G. Dietary red and processed meat intake and markers of adiposity and inflammation: the multiethnic cohort study. Journal of the American College of Nutrition 201736 378-385. (https://doi.org/10.1080/0731572 4.2017.1318317)

51 Lin WT, Kao YH, Sothern MS, Seal DW, Lee CH, Lin HY, Chen T \& Tseng TS. The association between sugar-sweetened beverages intake, body mass index, and inflammation in US adults. International Journal of Public Health 202065 45-53. (https://doi.org/10.1007/s00038-02001330-5)

52 Montonen J, Boeing H, Fritsche A, Schleicher E, Joost HG, Schulze MB, Steffen A \& Pischon T. Consumption of red meat and whole-grain bread in relation to biomarkers of obesity, inflammation, glucose metabolism and oxidative stress. European Journal of Nutrition 201352 337-345. (https://doi.org/10.1007/s00394-012-0340-6)

53 Casas R, Sacanella E \& Estruch R. The immune protective effect of the Mediterranean diet against chronic low-grade inflammatory diseases. Endocrine, Metabolic and Immune Disorders Drug Targets 201414 245-254. (https://doi.org/10.2174/1871530314666140922153350)

54 Koelman L, Markova M, Seebeck N, Hornemann S, Rosenthal A, Lange V, Pivovarova-Ramich $\mathrm{O} \&$ Aleksandrova K. Effects of high and low protein diets on inflammatory profiles in people with morbid obesity: a 3-week intervention study. Nutrients 202012 3636. (https:// doi.org/10.3390/nu12123636)

55 Markova M, Koelman L, Hornemann S, Pivovarova O, Sucher S, Machann J, Rudovich N, Thomann R, Schneeweiss R, Rohn S, et al. Effects of plant and animal high protein diets on immuneinflammatory biomarkers: a 6-week intervention trial. Clinical Nutrition 202039 862-869. (https://doi.org/10.1016/j. clnu.2019.03.019)

56 Mozaffarian D. Natural trans fat, dairy fat, partially hydrogenated oils, and cardiometabolic health: the Ludwigshafen risk and cardiovascular health study. European Heart Journal 201637 1079-1081. (https://doi. org/10.1093/eurheartj/ehv595)

57 Hosseini B, Berthon BS, Saedisomeolia A, Starkey MR, Collison A, Wark PAB \& Wood LG. Effects of fruit and vegetable consumption on inflammatory biomarkers and immune cell populations: a systematic literature review and meta-analysis. American Journal of Clinical Nutrition 2018108 136-155. (https://doi.org/10.1093/ajcn/nqy082)

58 Derry HM, Padin AC, Kuo JL, Hughes S \& Kiecolt-Glaser JK. Sex differences in depression: does inflammation play a role? Current Psychiatry Reports 201517 78. (https://doi.org/10.1007/s11920-0150618-5)

59 Daxer J, Herttrich T, Zhao YY, Vogel M, Hiemisch A, Scheuermann K, Körner A, Kratzsch J, Kiess W \& Quante M. Nocturnal levels of chemerin and progranulin in adolescents: influence of sex, body mass index, glucose metabolism and sleep. Journal of Pediatric Endocrinology and Metabolism 201730 57-61. (https://doi.org/10.1515/ jpem-2016-0378)

Received in final form 3 August 2021

Accepted 25 August 2021

Accepted Manuscript published online 25 August 2021 https://ec.bioscientifica.com https://doi.org/10.1530/EC-21-0273 (c) 2021 The authors Published by Bioscientifica Ltd

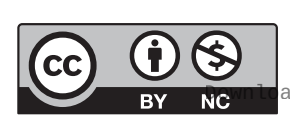

This work is licensed under a Creative Commons Attribution-NonCommercial 4.0 International License. ded from Bioscientifica.com at 04/26/2023 09:43:50AM 